\title{
Robust Distributed Kalman Filter for Wireless Sensor Networks with Uncertain Communication Channels
}

\section{Du Yong Kim and Moongu Jeon}

School of Information and Communication, Gwangju Institute of Science and Technology, Gwangju 500-712, Republic of Korea

Correspondence should be addressed to Moongu Jeon, mgjeon@gist.ac.kr

Received 28 November 2011; Revised 11 March 2012; Accepted 30 March 2012

Academic Editor: M. D. S. Aliyu

Copyright (C) 2012 D. Y. Kim and M. Jeon. This is an open access article distributed under the Creative Commons Attribution License, which permits unrestricted use, distribution, and reproduction in any medium, provided the original work is properly cited.

\begin{abstract}
We address a state estimation problem over a large-scale sensor network with uncertain communication channel. Consensus protocol is usually used to adapt a large-scale sensor network. However, when certain parts of communication channels are broken down, the accuracy performance is seriously degraded. Specifically, outliers in the channel or temporal disconnection are avoided via proposed method for the practical implementation of the distributed estimation over large-scale sensor networks. We handle this practical challenge by using adaptive channel status estimator and robust L1-norm Kalman filter in design of the processor of the individual sensor node. Then, they are incorporated into the consensus algorithm in order to achieve the robust distributed state estimation. The robust property of the proposed algorithm enables the sensor network to selectively weight sensors of normal conditions so that the filter can be practically useful.
\end{abstract}

\section{Introduction}

The estimation problem for a multisensor environment has been investigated for two decades [1-5]. Mainly two schemes are discussed to design the system: centralized fusion and distributed fusion.

Centralized fusion is a fusion architecture composed of one fusion center linked with multiple sensors. This architecture does not require a particular fusion rule; instead observations from multiple sensors are stacked as one sensor measurement whose size is very large. It is relatively easy to implement; however, communication bottle neck problems, sensor scheduling, and lack of flexibility are known as disadvantages [1]. Furthermore, if 
some communication channels between the central processor and sensor node are uncertain, it is difficult to manage in an adaptive manner.

On the other hand, decentralized fusion has been studied in the literature to tackle disadvantages of the centralized fusion [1]. Initial study of decentralized fusion is known as a decentralized Kalman filter in [2] which is mathematically equivalent to the centralized one. However, its usage is restricted only when sensor nodes are fully connected each other [2]. Namely, only one kind of network topology is allowed (e.g., all-to-all communication).

Based on this observation, the distributed fusion algorithm is suggested to consider issues in the practical implementation of the networked system [6]. For instance, the distributed fusion is required to resolve a network topology, measurement outliers, that is, sensor failure and limited communication bandwidth.

The network topology in multiagent systems has been actively investigated in control community and applied in the sensor network [3]. It was assumed that the network has fixed topology when sensor nodes are geometrically distributed and all communication channels operate in normal.

In parallel, regarding uncertain communication channel, single Kalman filter with intermittent observation gets explosive attentions in the network control system applications $[4,5]$. The main direction of conducted research was the stability analysis of the system under uncertain communication channels [4]. In this case, it is assumed that the communication channel uses TCP-like protocol that means that a packet is dropped based on its acknowledgement. Afterwards it has been extended for multisensor network systems [5]. The authors proposed the estimation algorithm using a sequential Kalman filtering using a set of recent observations collected from adjacent linked nodes. A tree topology is exploited for each node to understand the network topology of limited sensing range.

In another type of applications, however, we may not know the acknowledgement of the packet when the channel link between sensors is not reliable or changed due to the evolution of the network topology. For instance, mobile robots may change their topology based on the agents' location or there exit temporal disconnections in channels. In such cases, the packet arrival event should be estimated.

Previous research in data fusion and intermittent observation problem basically assumes that the noise statistic is known a priori, for example, Gaussian or bounded $[4,5,7]$. However, this assumption is often violated when outlier measurement happens.

The outlier can be originated from several practical challenges such as sensor failures, measurement outliers, or even intentional jamming. To solve this problem robust statistics has been investigated, for example, M-estimator [8]. L1-norm optimization was also considered as a solution because it is common that L1-norm optimization is robust against the outlier noise compared to the L2-norm optimization (e.g., Kalman filter). However, the use of L1norm is overlooked due to the computational complexity. With the help of advances in realtime convex optimization, L1-norm optimization is recently revisited and its application is rigorously investigated [9].

In this paper, we consider the estimation problem under multisensor environment with uncertain communication channels. Main tasks to solve this problem are (1) estimation of the channel status, (2) robust estimation to avoid outliers and uncertainty in communication channels, and (3) measurement fusion algorithm regarding to (1) and (2). The proposed work has a two-stage framework, that is, channel status estimation and L1-norm optimization-based outlier rejection. Note that to the author's knowledge there is no intensive research in the model-based state estimation problem (e.g., Kalman filtering) considering communication channel uncertainty over a large-scale sensor network. 
The remainder of the paper is organized as follows. Section 2 describes the problem formulation and the Kalman-consensus filter is introduced as a basic framework. Proposed algorithm is subsequently explained in Section 3 including channel status estimation, sparse optimization via L1-norm optimization, and the modified robust Kalman-consensus filter as an overall algorithm. A test example is provided to demonstrate the efficacy of the proposed algorithm in Section 4. Then, conclusion is made in Section 5.

\section{Problem Statement and Kalman-Consensus Filter}

\subsection{Problem Statement}

Consider a time-invariant linear system with Jump Markov measurement model as

$$
\begin{gathered}
x_{t+1}=A x_{t}+w_{t}, \\
y_{t}^{i}=C\left(\gamma_{t}^{i}\right) x_{t}+v_{t}^{i}+z_{t}^{i}, \quad i=1, \ldots, M,
\end{gathered}
$$

where $x_{t} \in \mathfrak{R}^{n}$ is the state (to be estimated) and $y_{t}^{i} \in \mathfrak{R}^{m}$ is the $i$ th measurement of a node in the network at time $t$, respectively. $A \in \mathfrak{R}^{n \times n}$ is the system matrix, and $C\left(\gamma_{t}^{i}\right) \in \mathfrak{R}^{m \times n}$ is the $i$ th measurement matrix of the node governed by a random latent variable $\gamma_{t}^{i}$ which describes the measurement mode $\gamma_{t}^{i}=1$ for packet received; $\gamma_{t}^{i}=0$, otherwise. $w_{t} \in \mathfrak{R}^{n}, v_{t}^{i} \in \mathfrak{R}^{m}$ and $z_{t}^{i} \in \mathfrak{R}^{m}$; are system noise, measurement noise, and sparse noise in the measurement, respectively. The sparse noise term $z_{t}^{i}$ models the outlier measurements whose magnitude is considerably large compared to the observation noise; thus it cannot be modeled as the standard Gaussian distribution. The process noise $w_{t}$ is independent identically distributed (i.i.d.) $N(0, Q)$ and the measurement noise $v_{t}^{i}$ is i.i.d. $N\left(0, R^{i}\right)$. Assume that the initial state $x_{0}$, the process noise, and the measurement noise are mutually uncorrelated each other.

Then, the main goal is to estimate the state $x_{0}$ given measurements from $M$ sensors up to time $t$, that is, $Y_{t}=\left\{y_{1: t^{\prime}}^{1}, \ldots, y_{1: t}^{M}\right\}$ where $y_{1: t}^{i}=\left\{y_{1}^{i}, \ldots, y_{t}^{i}\right\}$.

\subsection{Kalman-Consensus Filter}

In a large-scale sensor network, it is practically impossible that all the sensor nodes are fully connected each other. Therefore, there should be data fusion algorithm to adaptively aggregate sensor nodes into a globally reasonable estimate. In this study, we adopt a data fusion algorithm using a consensus protocol combined with Kalman filters of each node, called Kalman-consensus filter (KCF) [3]. Using a simple average consensus, individual decentralized Kalman filter called micro-Kalman filter communicates information with its neighbors and the state estimate. The flow of the information over the whole network is possible due to the graph Laplacian of the network topology. As illustrated in [3], even though the target state is partially observed with different groups of sensors and there is no fusion center, individual nodes agree with the converged estimate of the state.

Compared to other data fusion algorithms, KCF has advantages when error-cross covariance information is not available for pairs of sensor node. In addition, because the sensor network topology is incorporated in the data fusion algorithm, local information is propagated all over the network. 
In KCF framework, the communication topology between sensor nodes is represented by the directed graph $G=(V, E)$, where $V=\{1,2, \ldots, M\}$ denotes the sensor node set. The edge set $E \subset V \times V$ describes the communication links between each pair of nodes. The neighbor of sensor node $i$ is defined as $L_{i}: J_{i}=L_{i} \cup\{i\}$. KCF is implemented based on the information from of Kalman filter for each node and the consensus protocol to approach the global estimate as follows.

Assume that the latent variable $\gamma_{t}^{i}$ is known. The estimation of the latent variable will be given in following section. Then, contribution terms of the information Kalman filter are calculated for each node as follows:

$$
\begin{gathered}
u_{t}^{i}=\left(C\left(\gamma_{t}^{i}\right)\right)^{T}\left(R^{i}\right)^{-1} y_{t}^{i} \\
U_{t}^{i}=\left(C\left(r_{t}^{i}\right)\right)^{T}\left(R^{i}\right)^{-1} C\left(r_{t}^{i}\right) .
\end{gathered}
$$

Based on the known communication topology $G$, each node broadcasts its message $m_{t}^{i}=$ $\left(u_{t}^{i}, U_{t}^{i}, \bar{x}_{t}^{i}\right)$, where $\bar{x}_{t}^{i}$ is the priori state estimate of the sensor node $i$ and collects message $m_{t}^{r}=\left(u_{t}^{r}, U_{t}^{r}, \bar{x}_{t}^{r}\right)$ from its neighbors. Then, all the contribution terms are aggregated as $g_{t}^{i}=$ $\sum_{r \in J_{i}} u_{t}^{r}, S_{t}^{i}=\sum_{r \in J_{i}} U_{t}^{r}$.

With the aggregated contribution terms, each node calculates Kalman-consensus estimate using update step and prediction step as follows.

Update:

$$
\begin{gathered}
M_{t}^{i}=\left(\left(P_{t}^{i}\right)^{-1}+S_{t}^{i}\right)^{-1}, \\
\widehat{x}_{t+1}^{i}=\bar{x}_{t}^{i}+M_{t}^{i}\left(g_{t}^{i}-S_{t}^{i} \bar{x}_{t}^{i}\right)+\varepsilon \frac{M_{t}^{i}}{1+\left\|M_{t}^{i}\right\|} \sum_{r \in J_{i}}\left(\bar{x}_{t}^{r}-\bar{x}_{t}^{i}\right) .
\end{gathered}
$$

Prediction:

$$
\begin{gathered}
P_{t+1}^{i} \longleftarrow A_{t} M_{t}^{i} A_{t}^{T}+Q, \\
\bar{x}_{t+1}^{i} \longleftarrow A_{t} \widehat{x}_{t+1}^{i},
\end{gathered}
$$

where $\varepsilon$ is the discretization step size and $\|\cdot\|$ denotes the matrix norm.

$\mathrm{KCF}$ is easy to implement compared to other data fusion algorithms and scalable for large-scale networks [3]. However, it is assumed that the communication channel links are operating in normal which is often not the case in practical situations. When the topology has been changed, channel links are disconnected, or corrupted with outlier measurements, the performance of the consensus algorithm is not reliable anymore.

In this technical note, we propose the robust multisensor consensus estimator to avoid uncertainties in channel of the sensor network. We require three strategies to solve this problem: (1) channel status estimation, that is, mode estimation, (2) outlier rejection, and (3) data fusion. In the following section, we propose the robust Kalman-Consensus Filter based on the channel mode estimation, sparse optimization using L1-norm. 


\section{Main Results}

\subsection{Robust Kalman Filtering Using L1-Norm Optimization}

Kalman filter is known as an optimal estimator in linear Gaussian system. However, when the linear Gaussian assumption is violated in practice, additional modification is necessary. Because the outlier measurement is not able to be modeled as Gaussian, we model the outlier measurement as $z_{t}^{i}$ which is sparse. To alleviate the additional sparse noise, we adopt the robust Kalman filtering via sparse optimization using L1-norm in [9].

The standard Kalman filtering without the sparse noise is given as follows.

Prediction:

$$
\widehat{x}_{t \mid t-1}^{i}=A \widehat{x}_{t-1 \mid t-1}^{i} .
$$

Update:

$$
\hat{x}_{t \mid t}^{i}=\hat{x}_{t \mid t-1}^{i}+\Phi_{t \mid t}^{i}\left(C^{i}\right)^{T}\left(C^{i} \Phi_{t \mid t}^{i}\left(C^{i}\right)^{T}+R^{i}\right)^{-1}\left(y_{t}^{i}-C^{i} \widehat{x}_{t \mid t-1}^{i}\right)
$$

where $\Phi_{t \mid t}^{i}$ is the state error covariance. Here, we assume that the channel mode is completely known, that is, $C\left(\gamma_{t}^{i}\right) \triangleq C_{t}^{i}$. In the least square problem, Kalman filter is to minimize the cost function defined as

$$
\left(v_{t}^{i}\right)^{T}\left(R^{i}\right)^{-1} v_{t}^{i}+\left(x_{t}-\widehat{x}_{t \mid t-1}^{i}\right)^{T}\left(\Phi_{t \mid t}^{i}\right)^{-1}\left(x_{t}-\widehat{x}_{t \mid t-1}^{i}\right)
$$

subject to $y_{t}^{i}=C_{t}^{i} x_{t}+v_{t}^{i}$. The cost function of (3.1) is modified by adding regularization term considering the sparse noise $z_{t}^{i}$ as

$$
\left(v_{t}^{i}\right)^{T}\left(R^{i}\right)^{-1} v_{t}^{i}+\left(x_{t}-\widehat{x}_{t \mid t-1}^{i}\right)^{T}\left(\Phi_{t \mid t}^{i}\right)^{-1}\left(x_{t}-\widehat{x}_{t \mid t-1}^{i}\right)+\lambda\left\|z_{t}^{i}\right\|_{1}
$$

subject to $y_{t}^{i}=C_{t}^{i} x_{t}+v_{t}^{i}+z_{t}^{i}$. The minimization problem is solved using convex optimization. $\lambda$ is the regularization parameter.

The cost function of (3.4) is rewritten using residual $e_{t}^{i}=y_{t}^{i}-C_{t}^{i} \widehat{x}_{t \mid t-1}^{i}$ as

$$
J=\left(e_{t}^{i}-z_{t}^{i}\right)^{T} W\left(e_{t}^{i}-z_{t}^{i}\right)+\lambda\left\|z_{t}^{i}\right\|_{1}
$$

where $W=\left(I-C^{i} K_{t}^{i}\right)^{T}\left(R^{i}\right)^{-1}\left(I-C^{i} K_{t}^{i}\right)+\left(K_{t}^{i}\right)^{T}\left(\Phi_{t \mid t}^{i}\right)^{-1} K_{t}^{i} I$ is the identity matrix of appropriate dimension, and $K_{t}^{i}=\Phi_{t \mid t}^{i}\left(C_{t}^{i}\right)^{T}\left(C_{t}^{i} \Phi_{t \mid t}^{i}\left(C_{t}^{i}\right)^{T}+R^{i}\right)^{-1}$ is the Kalman gain. We solve the minimization of the cost function defined in (3.5) for the sparse noise as follows:

$$
\widehat{z}_{t}^{i}=\underset{z_{t}^{i}}{\arg \min } J
$$


Then the Kalman filter estimate is represented by

$$
\widehat{x}_{t \mid t}^{i}=\widehat{x}_{t \mid t-1}^{i}+K_{t}^{i}\left(e_{t}^{i}-\widehat{z}_{t}^{i}\right)
$$

Unlike the implementation in [9], our goal is to estimate the sparse noises and reject them from the measurements for the channel mode estimation in the next stage.

\subsection{Channel Mode Estimation}

Motivated by the work $[10,11]$, we try to estimate the channel mode $\gamma_{t}$ using moving horizon strategy. We denote the channel mode for the sensor node $i$ as $\gamma_{t}^{i}$ which is the discrete random variable. Assume that the evolution of the channel mode has the first-order Markov chain, its transition probability $\pi_{1,1}$ is the probability that the packet will arrive between time steps, and conversely $\pi_{0,1}$ represents the probability that the channel is switched off between time steps. Then, Bayesian update of the channel mode probability, that is, $\operatorname{Pr}\left(\gamma_{s}^{i} \mid y_{s-1}^{i}\right)$ in the moving horizon $[t-\Delta, t]$ is provided as follows.

For each measurement mode, $l=1 \rightarrow \gamma_{t}^{i}=1$, and $l=0 \rightarrow \gamma_{t}^{i}=0$.

Prediction:

$$
\operatorname{Pr}\left(r_{s}^{i} \mid y_{s-1}^{i}\right)=\pi_{1,1} \operatorname{Pr}\left(\gamma_{s-1}^{i} \mid y_{s-1}^{i}\right)+\pi_{0,1}\left(1-\operatorname{Pr}\left(r_{s-1}^{i} \mid y_{s-1}^{i}\right)\right)
$$

Update:

$$
\operatorname{Pr}\left(\gamma_{s}^{i} \mid y_{s}^{i}\right)=\frac{\Lambda_{s}^{i} \operatorname{Pr}\left(\gamma_{s}^{i} \mid y_{s-1}^{i}\right)}{1-\left(1-\Lambda_{s}^{i}\right) \operatorname{Pr}\left(\gamma_{s}^{i} \mid y_{s-1}^{i}\right)}, \quad s=t-\Delta, \ldots, t
$$

where the measurement likelihood of sensor node $i$ at time $s$ is defined as $\Lambda_{s}^{i} \triangleq y_{s}^{i}-C\left(\gamma_{s}^{i}\right) \widehat{x}_{s \mid s-1}^{i}$ and $\widehat{x}_{s \mid s-1}^{i}$ is the predicted state of local Kalman filter given in (3.1). Note that the recursion given in (3.8)-(3.9) is iterated in moving horizon $[t-\Delta, t]$ to obtain the channel mode estimate as $\widehat{\gamma}_{t}^{i}=1$, if $\operatorname{Pr}\left(\gamma_{t}^{i} \mid y_{t}^{i}\right)>$ Threshold 1 , or $\widehat{\gamma}_{t}^{i}=0$, if $\operatorname{Pr}\left(\gamma_{t}^{i} \mid y_{t}^{i}\right)<$ Threshold $_{0}$. In the channel mode probability calculation, we assume that the channel modes is not switched to other mode again at least within $\alpha$ steps. It is similar to the mode observability assumption given in [10]. Compared to the given assumption in [10], our assumption is not strict because we are not trying to distinguish the sequence of the mode in the horizon but to obtain the stable estimate of the current mode.

\subsection{Overall Algorithm}

In previous subsections we have discussed about robust Kalman filtering via L1-norm optimization and the channel mode estimation based on the channel mode probability. In this subsection, we combine two methods and suggest a robust data fusion algorithm to construct the overall implementation of our algorithm.

The overall flow of the proposed algorithm is displayed in Figure 1. The dynamic state process is observed from multisensors. To efficiently reject the sparse measurement outliers, 


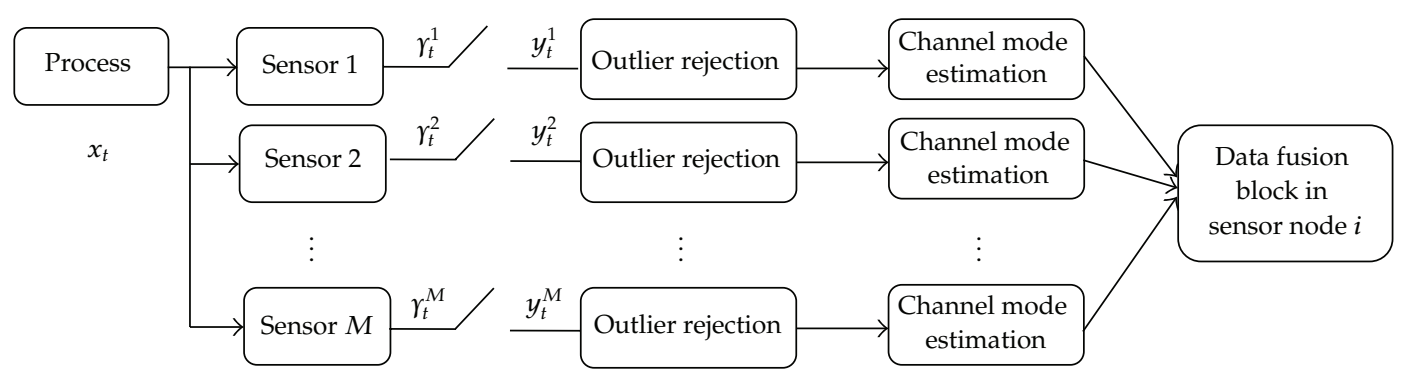

Figure 1: Overall flow of the proposed algorithm.

L1-norm optimization is subsequently utilized. After trimming multiple measurements by rejection of outliers, we estimate the channel mode of each sensor node and finally fuse the set of estimate and measurement based on the consensus protocol which is explained in (2.2)-(2.4). According to the overall flow of the proposed algorithm described in Figure 1, we summarize the robust distributed fusion consensus filter in Algorithm 1.

Remark 3.1. Consider that error convergence of the algorithm L1-norm optimization and measurement mode estimation are main concerns. When we modified Kalman filtering update step with L1-norm optimization then, it is not Gaussian estimate anymore. So, it is not straightforward to readily analyze the error convergence in modified Kalman filtering. Therefore, it is remained as a future work for ours.

Remark 3.2. Considering the measurement mode observability, we follow the idea similar to [10] that there is a minimum dwell time of the measurement mode switching. Thus, we set the horizon window size $\Delta$ as the minimum dwell time of the measurement mode switching. In practice, this value is the design parameter for the network. However, the value of the delta is not that sensitive to the minimum dwell time of the channel, that is, tuning of the delta is not that sensitive. In addition, in cases where frequent switching happens, we regard it as outlier measurement and it will be handled via robust Kalman filtering step in L1-norm optimization.

Remark 3.3. In the experiment, we set the horizon size as 5 when the switching probability is $\pi_{1,0}=\pi_{0,1}=0.05$. In our experiment, if the mode is switched within $\tau$ step which is less than the predefined horizon size $\Delta$, then the performance of the channel mode estimator is not reliable. Thus, as already explained in Remark 3.1, frequent switching would be considered as permanent channel link break down. However, rather fast but not abnormally frequent switching can be handled via switching Kalman filters (e.g. interacting multiple model filter (IMM filter)).

\section{Illustrative Example}

In this section, we test the efficacy of the proposed algorithm with the state estimation problem using a large-scale sensor network.

Given the target dynamics of a circular movement [3]

$$
x_{t+1}=A x_{t}+B w_{t},
$$


Given $\sum_{t \mid t-1}^{i}$, error covariance, $\widehat{x}_{t \mid t-1}^{i}$, previous estimate, consensus update parameter $\varepsilon$, and the window size $\Delta$.

1. Obtain measurement $y_{t}^{i}=C\left(\gamma_{t}^{i}\right) x_{t}+v_{t}^{i}+z_{t}^{i}, i=1, \ldots, N$.

2. For each measurement solve L1-norm optimization problem, reject outliers as given in (3.5) and then obtain the trimmed measurements: $\widehat{y}_{t}^{i}=y_{t}^{i}-\widehat{z}_{t}^{i}$.

3. Calculate the mode probability $\operatorname{Pr}\left(\gamma_{t}^{i} \mid \widehat{y}_{t-\Delta: t}^{i}\right)$.

Given $\operatorname{Pr}\left(\gamma_{t-\Delta}^{i} \mid \widehat{y}_{t-\Delta}^{i}\right)$,

For $s=t-\Delta: t$

Evaluate measurement likelihood for $\widehat{y}_{s}^{i}$.

Evaluate the Bayesian recursion (3.8)-(3.9).

End

Decide the channel mode $\widehat{\gamma}_{t}^{i}$ using threshold testing.

4. Compute contribution term of information state and matrix such that

$$
\begin{gathered}
u_{t}^{i}=\left(C_{t}^{i}\left(\widehat{\gamma}_{t}^{i}\right)\right)^{T}\left(R^{i}\right)^{-1} \widehat{y}_{t \prime}^{i} \\
U_{t}^{i}=\left(C_{t}^{i}\left(\widehat{\gamma}_{t}^{i}\right)\right)^{T}\left(R^{i}\right)^{-1} C_{t}^{i}\left(\widehat{\gamma}_{t}^{i}\right) .
\end{gathered}
$$

5. Broadcast message $m_{t}^{i}=\left(u_{t}^{i}, U_{t}^{i}, \widehat{x}_{t \mid t-1}^{i}\right)$ to neighbors in $L_{i}$.

6. Collect messages $m_{t}^{r}=\left(u_{t}^{r}, U_{t}^{r}, \widehat{x}_{t \mid t-1}^{r}\right)$ from neighbors.

7. Aggregate the information states and matrices of neighbors including node $i: J_{i}=L_{i} \cup\{i\}$ :

$$
g_{t}^{i}=\sum_{r \in J_{i}} u_{t}^{r}, \quad S_{t}^{i}=\sum_{r \in J_{i}} U_{t}^{r} .
$$

8. Compute the Kalman-Consensus estimate:

$$
\begin{gathered}
\left(M_{t}^{i}\right)^{-1}=\left(\Phi_{t \mid t-1}^{i}\right)^{-1}+S_{t}^{i} \\
\widehat{x}_{t \mid t}^{i}=\widehat{x}_{t \mid t-1}^{i}+M_{t}^{i}\left(g_{t}^{i}-S_{t}^{i} \hat{x}_{t \mid t-1}^{i}\right)+\varepsilon \frac{M_{t}^{i}}{1+\left\|M_{t}^{i}\right\|} \sum_{r \in J_{i}}\left(\widehat{x}_{t \mid t-1}^{r}-\widehat{x}_{t \mid t-1}^{i}\right) . \\
\text { Prediction stage } \\
\Phi_{t+1 \mid t}^{i} \longleftarrow A M_{t}^{i} A^{T}+Q, \\
\widehat{x}_{t+1 \mid t}^{i} \longleftarrow A \widehat{x}_{t \mid t}^{i} .
\end{gathered}
$$

Algorithm 1: Robust distributed fusion algorithm for node $i$.

where $A_{0}=2\left[\begin{array}{cc}0 & -1 \\ 1 & 0\end{array}\right], B_{0}=5^{2} I_{2}, A=I_{2}+\varepsilon A_{0}+\left(\varepsilon^{2} / 2\right) A_{0}^{2}+\left(\varepsilon^{2} / 6\right) A_{0}^{3}$, and $B=\varepsilon B_{0}$. In addition, $I_{2}$ is a $2 \times 2$ identity matrix which is a discretized model with a step size $\varepsilon=0.015$, and the initial position and uncertainty are $x_{0}=(15,-10)^{T}$ and $P_{0}=10 I_{2}$, respectively. A moving target having a circular motion can then be observed via the large-scale sensor network of 100 sensor nodes as displayed in Figure 2. Here, the sensor nodes measure the target position with uncertain communication channel links between nodes as

$$
y_{t}^{i}=C\left(\gamma_{t}^{i}\right) x_{t}+v_{t}^{i}, \quad t=0,1, \ldots, i=1, \ldots, 100,
$$

where either

$$
C\left(\gamma_{t}^{i}\right)=\left\{\begin{array}{ll}
{\left[\begin{array}{ll}
1 & 0
\end{array}\right],} & \text { if } \gamma_{t}^{i}=1, \\
{\left[\begin{array}{ll}
0 & 0
\end{array}\right],} & \text { if } \gamma_{t}^{i}=0,
\end{array} \text { or } \quad C\left(\gamma_{t}^{i}\right)= \begin{cases}{\left[\begin{array}{ll}
0 & 1
\end{array}\right],} & \text { if } \gamma_{t}^{i}=1, \\
{\left[\begin{array}{ll}
0 & 0
\end{array}\right],} & \text { if } \gamma_{t}^{i}=0 .\end{cases}\right.
$$




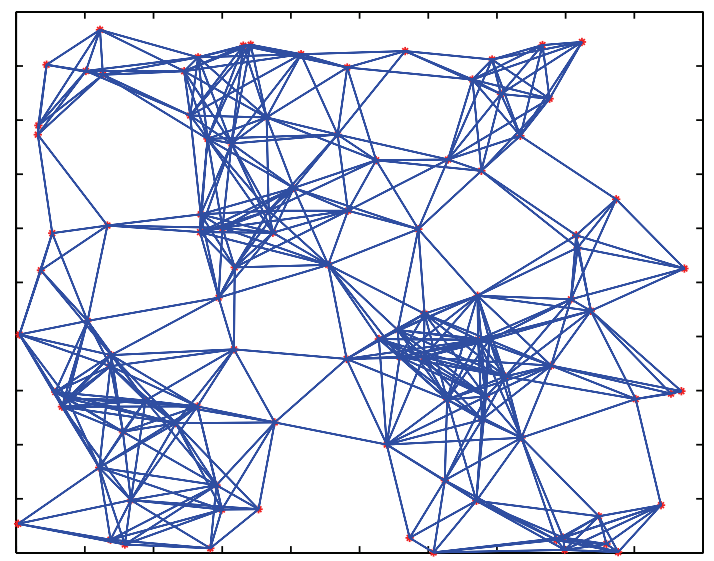

Figure 2: A large-scale sensor network with 100 nodes.

In the observation model, individual sensor measures either $x$-position or $y$-position. For each sensor node we model the channel mode latent variable $\gamma_{t}^{i}$ that describes the channel condition. To simulate the true observation mode for each node, the mode switch in the communication channel link is modeled as $\operatorname{Pr}\left(\gamma_{t}^{i}=0\right) \equiv \operatorname{Pr}(u(0,1)<0.01)$, where $u(0,1)$ is a uniform random distribution. We also model the evolution of channel mode variable as the first-order Markov chain. In this case, the transition probability between modes is given a priori. The observation noise for each sensor is white Gaussian noise with $v_{t}^{i} \sim N\left(0,30^{2} \sqrt{i}\right)$. In addition, sparse noises are generated with the probability 0.05 , whose magnitude is 10 times larger than that of the measurement noise.

\subsection{Comparison with KCF}

In the experiment we compare our proposed algorithm with standard KCF. Figure 3 simply and clearly demonstrates that our algorithm is robust when there are practical challenges in the network.

To show more clearly the robustness against the outliers, we select one sensor node experiment. That is because in KCF framework, certain amount of uncertainty can be aggregated via consensus update. The comparison of estimated trajectory with the ground truth is given in Figure 4. Measurements are also displayed with outliers to show that the proposed estimation in sensor node considerably improved mean square error (MSE) as illustrated in Figure 5.

\subsection{Comparison with Switching Kalman Filter}

As mentioned in Remark 3.3, rather fast switching of the channel mode can be handled more accurately via the IMM filter that is known as switching Kalman filter [12]. From our experiments, the proposed method (i.e., observation mode estimation via moving horizon strategy) is more accurate when the actual switching of the channel occurs in more than $\Delta$ steps. It means that the moving horizon strategy guarantees us the stable estimate of the observation mode when the minimum dwell time assumption is held as described in Section 3.2. On the other hand, the IMM filter shows us slightly increased errors in this case 


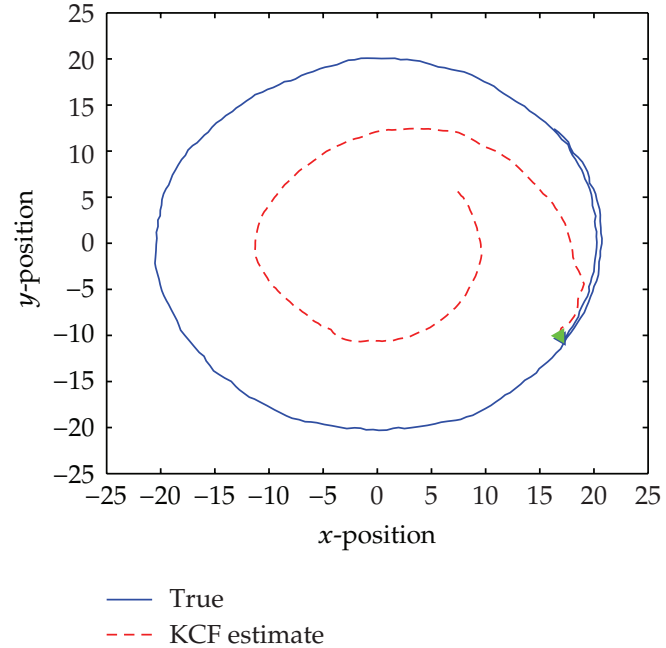

(a)

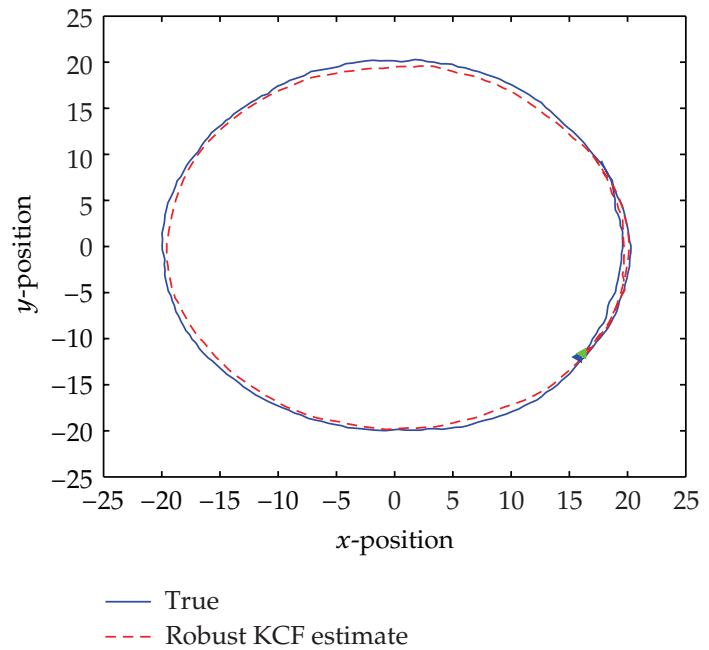

(b)

Figure 3: Comparison of estimated trajectories ((a) ground truth with $\mathrm{KCF}$, (b) truth with robust $\mathrm{KCF})$.

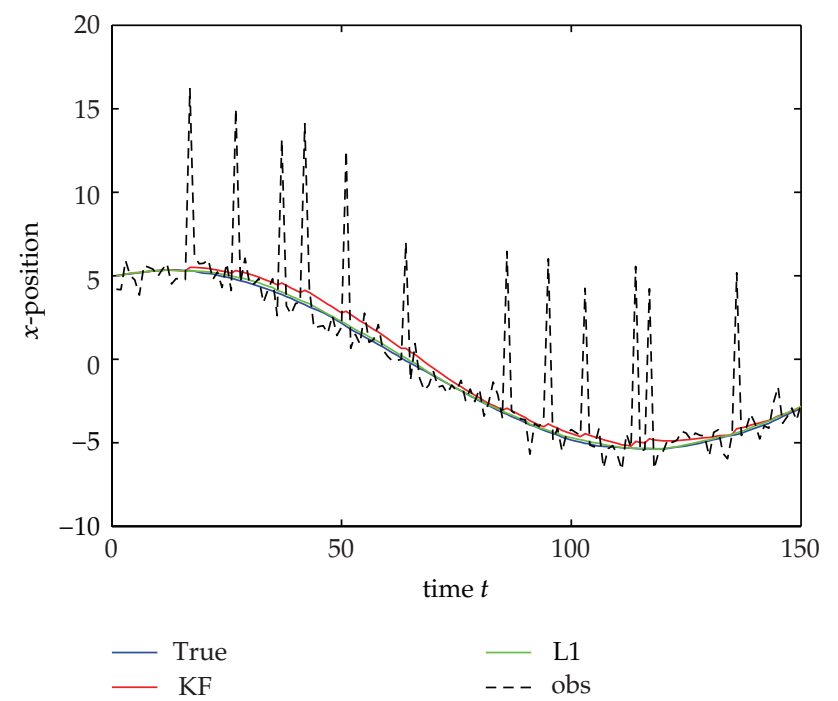

Figure 4: Comparison of estimated trajectories with measurements for single node (KF: micro KF; L1: proposed, obs: measurements).

because the IMM does not determine the exact mode as 1 or 0 . Instead, the mode probability is calculated and utilized for weighted averaging. However, the IMM filter is more robust in cases where mode switching frequently occurs. That is because there is no minimum dwell time assumption in the IMM filter.

In terms of computational complexity, the IMM filter is implemented using two parallel Kalman filters for each observation mode, that is, the complexity is approximately $O\left(2 n^{2}|E|+n^{2} N\right)$, where $n$ is the dimension of the state, $N$ is the number of nodes, and $|E|$ is the number of edge (e.g., links) in the network, as already mentioned in Section 2. In contrast, 


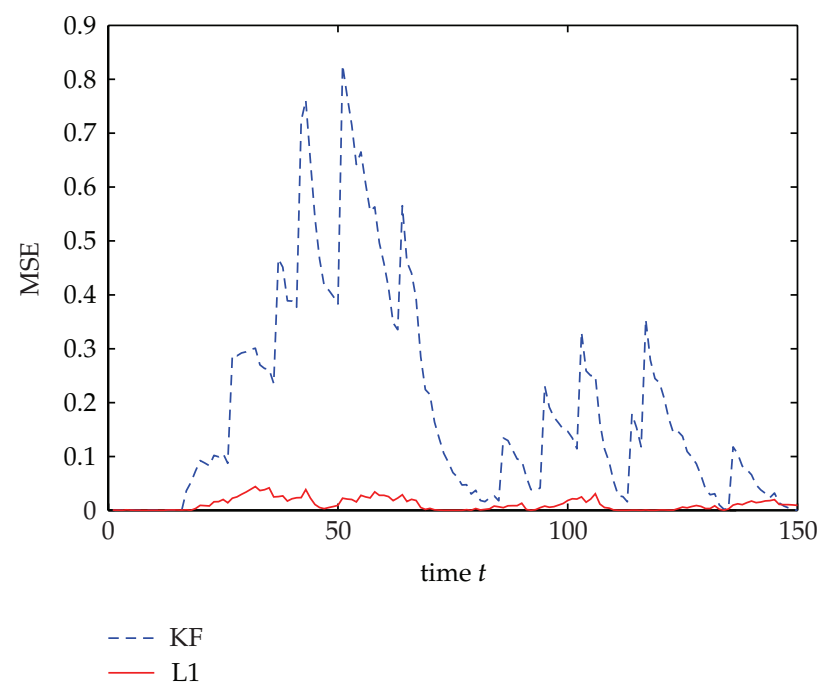

Figure 5: Comparison of MSE.

the proposed algorithm requires a recursion for mode estimation within a horizon window; thus, the complexity is approximated as $O\left(n \Delta|E|+n^{2} N\right)$. Therefore, the proposed algorithm is less complex than the IMM algorithm. Note that the complexity of the Kalman filter is $O\left(n^{2}\right)$.

\section{Conclusion}

In this paper we propose a novel distributed data fusion algorithm that is robust against outlier measurements and channel uncertainty. Outliers are rejected from the L1-norm optimization algorithm and the channel uncertainty is reduced using the measurement mode estimation algorithm. For the implementation in a large-scale sensor network, we adopt the KCF framework and test the framework with an object state estimation problem. Results successfully demonstrate that the proposed framework is able to handle practical challenges.

\section{Acknowledgments}

This work was supported in part by GIST Systems Biology Infrastructure Establishment Grant by the Center for Distributed Sensor Network at GIST and by the Unmanned Technology Research Center at KAIST, originally funded by DAPA, ADD, and Republic of Korea.

\section{References}

[1] D. L. Hall and J. Llinas, "An introduction to multisensor data fusion," Proceedings of the IEEE, vol. 85, no. 1, pp. 6-23, 1997.

[2] H. F. Durrant-Whyte, B. Y. S. Rao, and H. Hu, "Toward a fully decentralized architecture for multisensor data fusion," in Proceedings of the IEEE International Conference on Robotics and Automation, pp. 1331-1336, May 1990. 
[3] R. Olfati-Saber, "Distributed Kalman filtering for sensor networks," in Proceedings of the 46th IEEE Conference on Decision and Control (CDC '07), pp. 5492-5498, December 2007.

[4] B. Sinopoli, L. Schenato, M. Franceschetti, K. Poolla, M. I. Jordan, and S. S. Sastry, "Kalman filtering with intermittent observations," IEEE Transactions on Automatic Control, vol. 49, no. 9, pp. 1453-1464, 2004.

[5] L. Shi, "Kalman filtering over graphs: theory and applications," IEEE Transactions on Automatic Control, vol. 54, no. 9, pp. 2230-2234, 2009.

[6] P. Alriksson and A. Rantzer, "Experimental evaluation of a distributed Kalman filter algorithm," in Proceedings of the 46th IEEE Conference on Decision and Control (CDC '07), pp. 5499-5504, December 2007.

[7] M. Epstein, L. Shi, and R. M. Murray, "An estimation algorithm for a class of networked control systems using UDP-like communication schemes," in Proceedings of the 45th IEEE Conference on Decision and Control (CDC '06), pp. 5597-5603, December 2006.

[8] P. J. Huber, Robust Statistics, John Wiley \& Sons, New York, NY, USA, 2nd edition, 1981, Wiley Series in Probability and Mathematical Statistics.

[9] J. Mattingely and S. Boyd, "Real-time convex optimization in signal processing," IEEE Signal Processing Magazine, vol. 27, no. 3, pp. 50-61, 2010.

[10] A. Alessandri, M. Baglietto, and G. Battistelli, "A maximum-likelihood Kalman filter for switching discrete-time linear systems," Automatica, vol. 46, no. 11, pp. 1870-1876, 2010.

[11] D. Y. Kim, J. H. Yoon, Y. H. Kim, and V. Shin, "Distributed information fusion filter with intermittent observations," in Proceedings of the 13th Conference on Information Fusion (FUSION '10), July 2010.

[12] H. A. P. Blom and Y. Bar-Shalom, "Interacting multiple model algorithm for systems with Markovian switching coefficients," IEEE Transactions on Automatic Control, vol. 33, no. 8, pp. 780-783, 1988. 


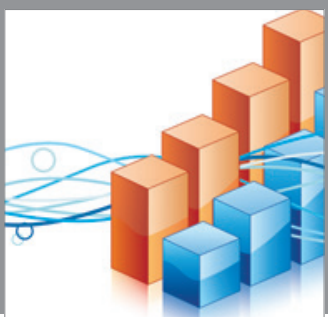

Advances in

Operations Research

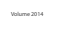

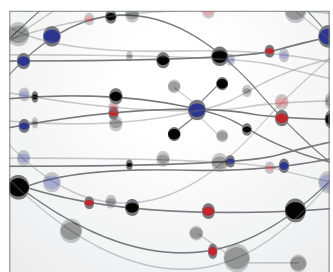

\section{The Scientific} World Journal
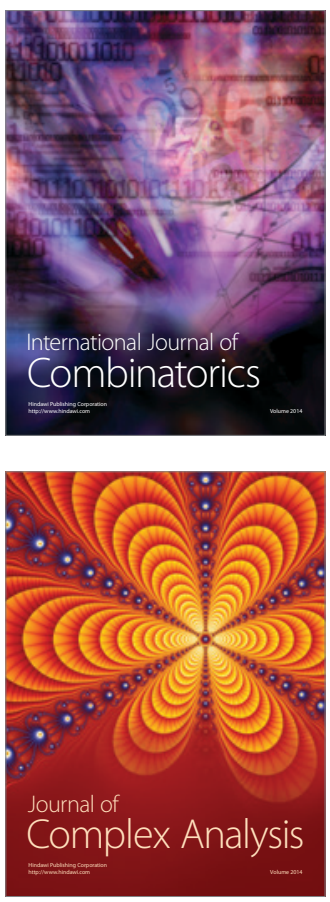

International Journal of

Mathematics and

Mathematical

Sciences
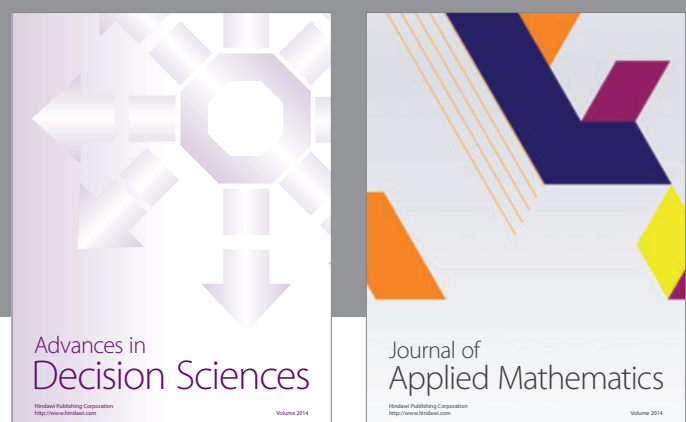

Journal of

Applied Mathematics
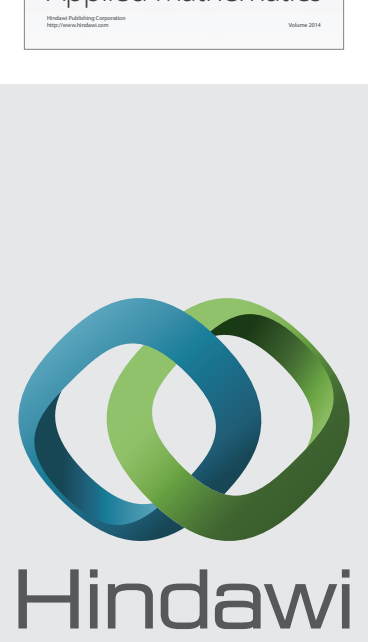

Submit your manuscripts at http://www.hindawi.com
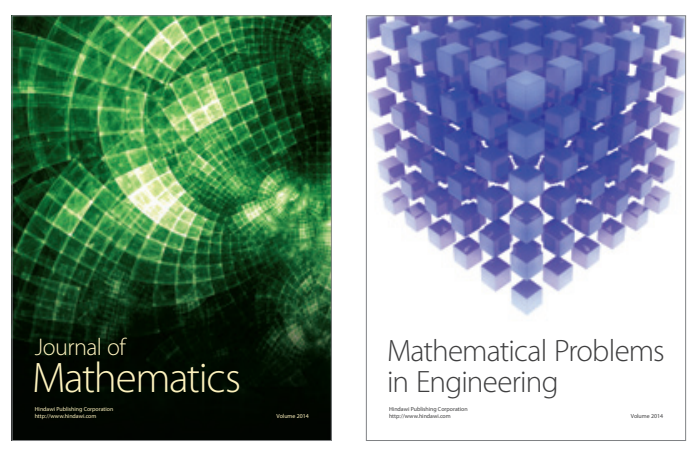

Mathematical Problems in Engineering
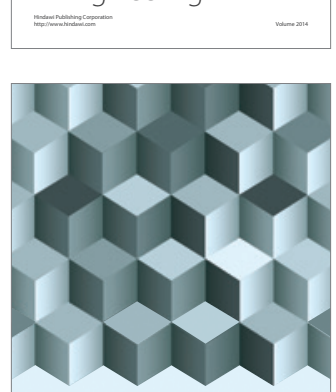

Journal of

Function Spaces
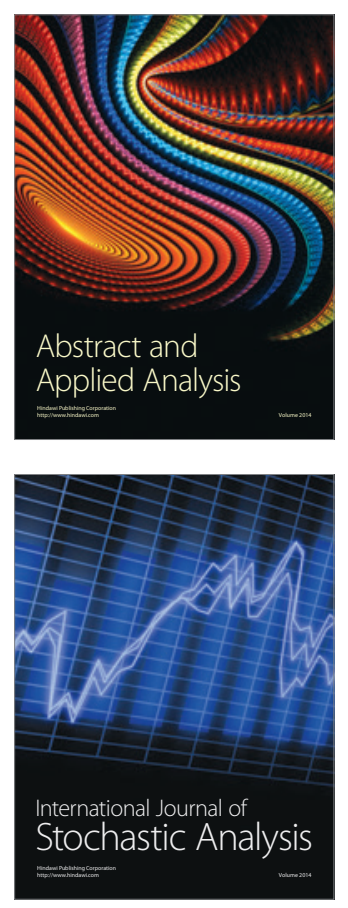

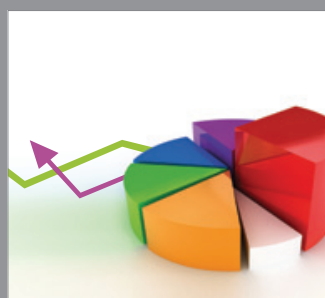

ournal of

Probability and Statistics

Promensencen
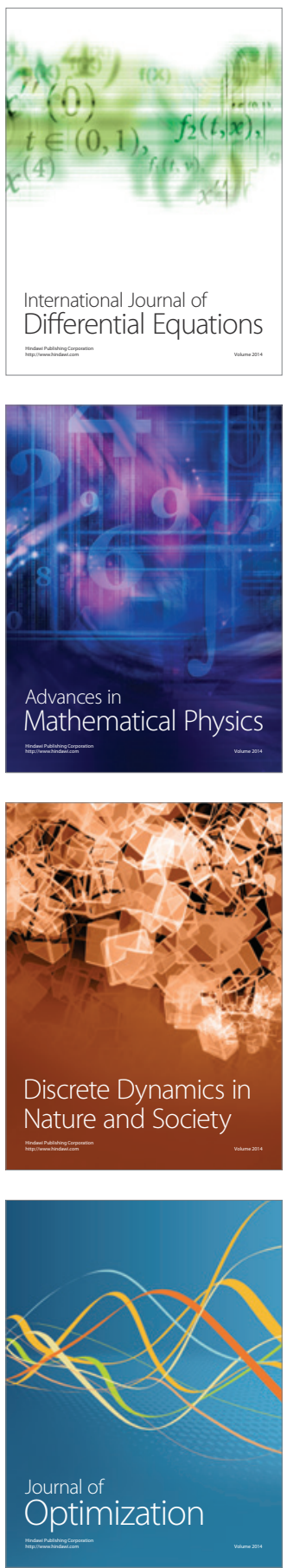